\title{
NOISY IMAGE SEGMENTATION USING A SELF-ORGANIZING MAP NETWORK
}

\author{
Saleh Gorjizadeh ${ }^{1}$, Sadegh Pasban², Siavash Alipour ${ }^{3}$
}

1 Student in the Department of Computer Engineering, Islamic Azad University, Sari, Iran, e-mail: Gorjizadeh. saleh@gmail.com

2 Department of Computer Engineering at Birjand University, Birjand, Iran, e-mail: sadegh.info@gmail.com

${ }^{3}$ Department of Electrical and Electronic Engineering at Malek-Ashtar University of Technology, Tehran, Iran, e-mail: siavash.alipur@gmail.com

Received: 2015.04.07

Accepted: 2015.05.08

Published: 2015.06.01

\begin{abstract}
Image segmentation is an essential step in image processing. Many image segmentation methods are available but most of these methods are not suitable for noisy images or they require priori knowledge, such as knowledge on the type of noise. In order to overcome these obstacles, a new image segmentation algorithm is proposed by using a self-organizing map (SOM) with some changes in its structure and training data. In this paper, we choose a pixel with its spatial neighbors and two statistical features, mean and median, computed based on a block of pixels as training data for each pixel. This approach helps SOM network recognize a model of noise, and consequently, segment noisy image as well by using spatial information and two statistical features. Moreover, a two cycle thresholding process is used at the end of learning phase to combine or remove extra segments. This way helps the proposed network to recognize the correct number of clusters/segments automatically. A performance evaluation of the proposed algorithm is carried out on different kinds of image, including medical data imagery and natural scene. The experimental results show that the proposed algorithm has advantages in accuracy and robustness against noise in comparison with the well-known unsupervised algorithms.
\end{abstract}

Keywords: image segmentation; unsupervised algorithms; noise; statistical features; SOM neural network.

\section{INTRODUCTION}

This Image segmentation is the process of image division into regions with similar attributes $[1,2]$. It is an important step in image analysis chain with applications to satellite images, such as locating objects (roads, forests, etc.), face recognition systems, and Medical Imaging [3]. The objective of segmentation is to simplify and/or change the representation of an image into something that is more meaningful and easier to analyze [3]. The result of image segmentation is a set of regions that collectively cover the entire image, or a set of contours extracted from the image. Each pixel in a region is similar with respect to some characteristic or computed property, such as color, intensity, or texture.

Image segmentation can be considered to be a kind of clustering, which clusters similar pixels into same group. Clustering by supervised and unsupervised learning [4] is considered as the most popular segmentation technique. Until recently, most of the segmentation methods and approaches are supervised such as Maximum Posteriori (MAP) [5] or Maximum Likelihood (ML) [6] with an average efficiency rate of about $85 \%[7,8]$. In the supervised methods a priori knowledge is needed to get a successful segmentation process and sometime the required information may not be available. 
On the other hand, in unsupervised technique inherent features extracted from the image is used for the segmentation. Unsupervised segmentation based on clustering includes K-means, Fuzzy C-Means (FCM) and ANN. K-means algorithm is a hard segmentation method because it assigns a pixel to a class or it does not [4]. FCM uses a membership function so that a pixel can belongs to several clusters having different degree. One important problem of these two clustering methods is that the clustering numbers must be known beforehand. ANN can change their responses according to the environmental conditions and learn from experience. Self-Organizing Map (SOM) $[9,10]$ or Kohonen's Map is an unsupervised ANN that uses competitive learning algorithm. The SOM features are very useful in data analysis and data visualization, which makes it an important tool in image segmentation [4]. Although the use of SOM in image segmentation is well reported in the literature $[9,11]$, its application under noisy condition is not widely known.

This paper proposes a developed self-organizing-map to segment images under noisy cases with high performance. Using two Statistical features, mean and median, calculated according to a block of pixels, and all pixels in this block as part of SOM input learning data for each pixel, leads to a suitable segmentation with respect to noise.

\section{SELF-ORGANIZING MAP}

The SOM introduced by Kohonen [12], is an unsupervised learning neural network. SOM projects a high dimensional space to a one or two dimensional discrete lattice of neuron units. Each node of the map is defined by a vector Wij, whose elements are adjusted during the training. An important feature of this neural network is its ability to process noisy data. The map preserves topological relationships between inputs in a way that neighboring inputs in the input space are mapped to neighboring neurons in the map space [13].

In SOM, the neurons are arranged into the nodes of a lattice that is shown in Figure 1 [14]. The basic SOM model consists of two layers. The first layer contains the input nodes and the second one contains the output nodes. The output nodes are arranged in a two dimensional grid $[15,16]$. Every input is connected extensively to every output via adjustable weights [17].
Best matching unit and finding the winner neuron determined by the minimum Euclidean distance to the input. Let $x$ be the input and $W_{i j}$ be the weight vector to the nodes. Vector $x$ is compared with all the weight vectors. The smallest Euclidian distance $\left(d_{i j}\right)$ is defined as the bestmatching unit (BMU) or winner node.

$$
d_{i j}=\min \left\|x(t)-w_{i j}\right\|
$$

Adjustment of the weight vector for the winning output neuron and its neighbors are calculated as followed:

$$
\begin{gathered}
w_{i j}(t+1)=w_{i j}(t)+\alpha(t)\left[x(t)-w_{i j}(t)\right], \quad i \in N_{c} \\
w_{i j}(t+1)=w_{i j}(t), i \notin N_{c}
\end{gathered}
$$

Where for time $\mathrm{t}$, and a network with $\mathrm{n}$ neurons: $\alpha$ is the gain sequence $(0<\alpha<1)$ and $N_{c}$ is the neighborhood of the winner $\left(1<N_{c}<\mathrm{n}\right)$.

The basic training algorithm is quite simple:

1) Each node's weights are initialized.

2) Vector is chosen at random from the set of training data.

3) Every node is examined to calculate which node's weight is most alike the input vector. The winning node is commonly known as the Best Matching Unit

4) Then the neighborhood of the BMU is calculated. The amount of neighbors decreases over time.

5) Update weights to node and neighbors according to equation (2).

6) If $N_{c} \neq 0$ then repeat step 2.

\section{PROPOSED SOM METHOD}

Although normal SOM has sufficient result through its features such as learning capability from examples, generalization ability, and nonparametric estimation, it suffers from two main problems. First, it is highly dependent on the training data representatives [18], especially in noisy situations. So, choosing a suitable training data is one of the most important parts in SOM. Second, Normal SOM cannot recognize segment or cluster's numbers automatically. This section proposes a new SOM algorithm to segment input images in both noisy and non-noisy cases. This algorithm consists of two steps:

First, training data for each pixel are chosen via a pixel with its block values and two statistical features as well. A block of each pixel is made by its spatial neighbors. Moreover, two statistical features, median and mean values, which are 
computed according to intensity values of the block, are employed to recognize a model of noises. Median value helps the proposed SOM model to identify salt \& pepper noise and means value help it to identify Gaussian noise too. In fact, the proposed SOM does take spatial information and two statistical features into account in order to recognize model of noise and consequently segment noisy image as well.

Second, we use a maximum cluster number instead of a predefined number of SOM output cluster. In addition, a two cycle thresholding process is used at the end of SOM learning phase to remove the unnecessary cluster. In fact, by using these two thresholds, we would not need to a prior knowledge about the number of clusters in SOM method. These two cycles process are describe as below.

In the first cycle, we remove clusters which their data numbers are less than a specific threshold, $T 1$, (clusters with few pixels). $T 1$ is computed via the number of image pixels. Then, the data whose clusters are removed will put into a cluster according to the nearest Euclidean distance between the data and center of clusters. To reduce over segmentation problem, in the second cycle, two clusters are combined if the distance between their cluster centers is less than a predefined threshold $T$. Figure 2 shows a scheme of proposed method.

\section{EXPERIMENTAL RESULT}

This section presents several results of the simulation on the segmentation of medical and famous public Berkeley segmentation dataset (Fig. 3). These results illustrate the ideas presented in the previous section. Three images are shown in Figure 1. The first image is a Brain MRI that consists four objects: CSF, white matter, grey matter and background from [19]. The second is

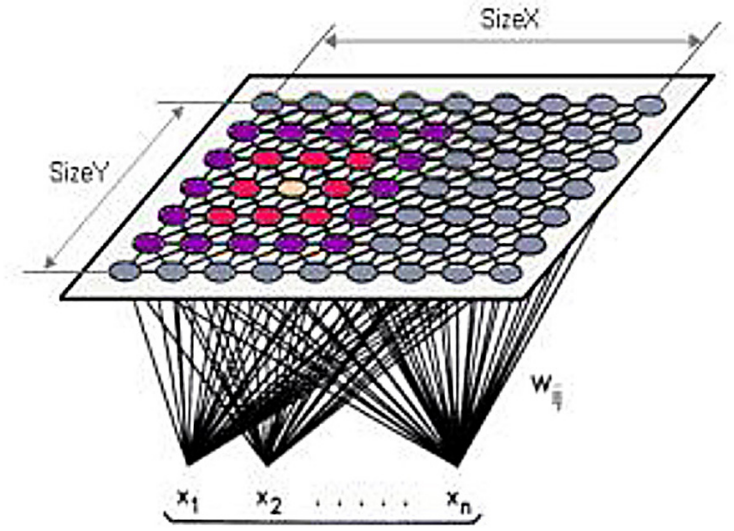

Fig. 1. Mapping of feature vector to the output

an X-ray image of a vessel with intensity inhomogeneity which consists of two objects: vessel and background from [20]. The target is to eliminate the vessel. The third is a camera man image which consists of three regions from [21].

These images are commonly used in papers [20-23] for image segmentation purposes and the algorithms compared have employed these images in their experiments. The original images are stored in grayscale space which take 8 bits and have the intensity range from 0 to 255 . We have to cluster pixels of each image by our new algorithm and compare the result with K-Mean [24], FCM [25] and normal SOM [12] methods on image segmentation. In this paper, T1 is equal to $5 \%$ of image pixel's numbers, $T 2=75$. Moreover, the maximum number of clusters is set 12 for experiments.

Figures 4 and 5 present the simulation results of three images corrupted with $15 \%$ and $25 \%$ salt and pepper noise respectively. Moreover, to show that the proposed method is robust to Gaussian noise, the next experiment is designed. We add the Gaussian noise with mean $=0$, variance $=0.25$ to the three images present in Figure 3 and presented the results in Figure 6. In the first column

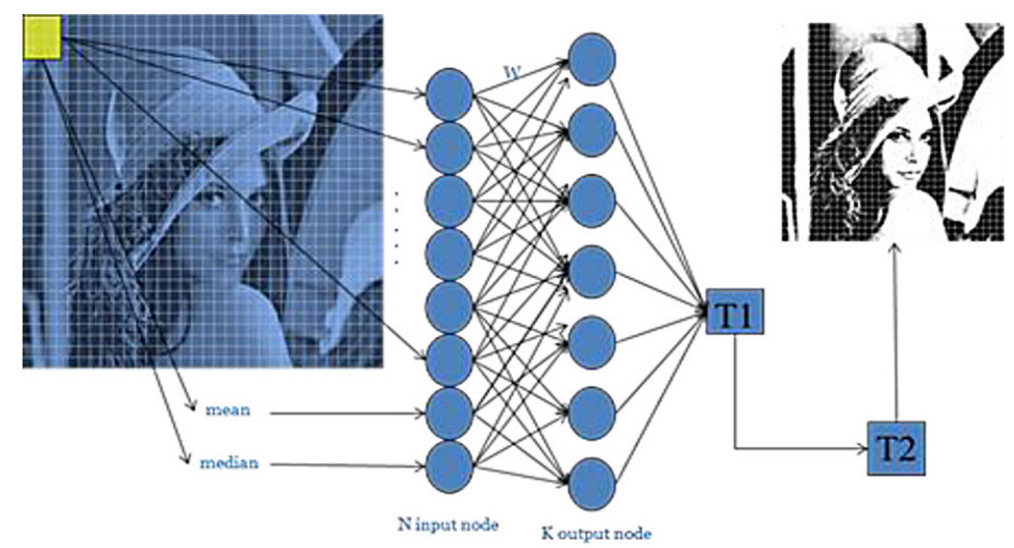

Fig. 2. The proposed model of segmentation with developed SOM 

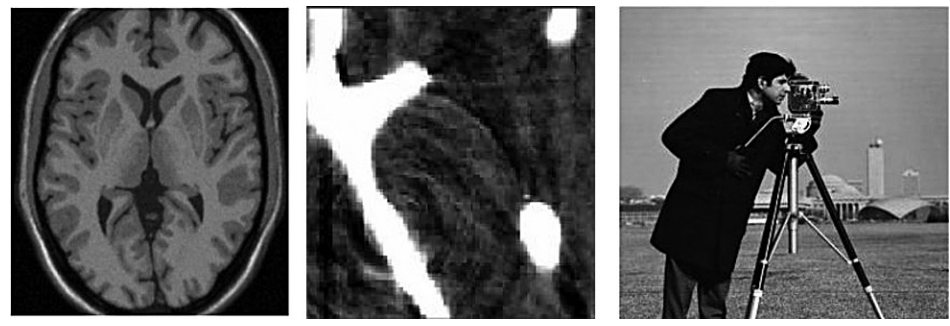

Fig. 3. Data set used in the experiment
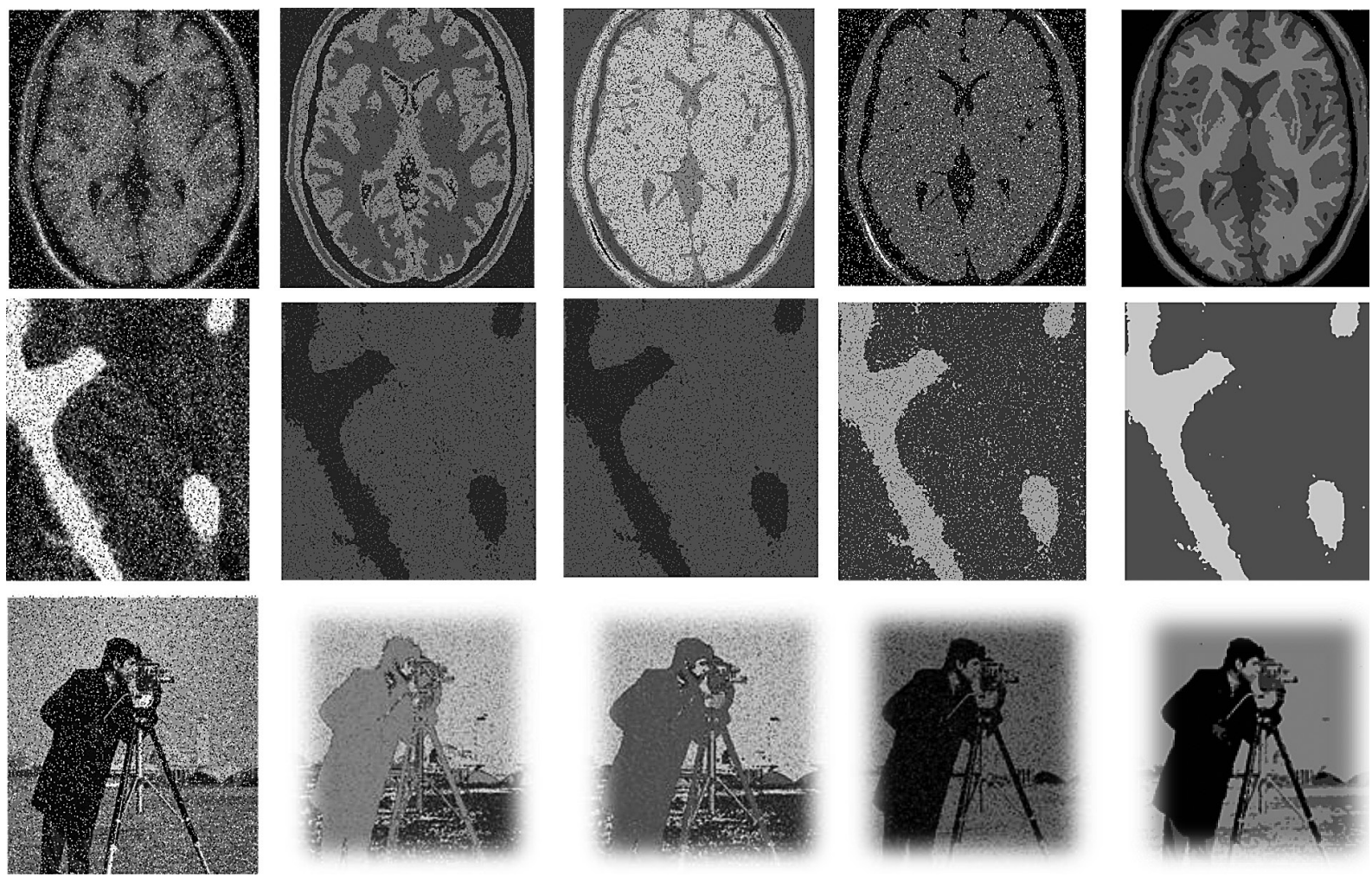

Fig. 4. The segmentation result of the three images contaminated with $15 \%$ salt and pepper noise in the first column was generated by the K-Mean (the second column), the FCM (the third column), the standard SOM (the fourth column) and the proposed SOM (fifth column)

of Figures 4-6 are the input noisy images; in the second, the third and the fourth columns are the segmentation results by the K-Mean, FCM and normal SOM respectively. The fifth column shows the results of the proposed SOM method.

From Figures 4-6, it can be seen that the proposed method performs well for all images. However, the rest fails in most situations. The reason is that the proposed SOM attempts to adjust weight vectors of the winning output neuron through spatial and statistical information of each pixel. Consequently, it shows lower susceptibility to noise. But, the standard SOM algorithm and also K-Mean and FCM methods are only concerned with intensity information and this information is changed by noise. Consequently, these algorithms are sensitive with respect to noise.

\section{CONCLUSION}

In this paper, we have presented a robust and effective approach for the segmentation of natural and medical images corrupted by different type of noise. For the segmentation of noisy images, the proposed approach utilized a SOM-based clustering with spatial and statistical information which are computed based on a block of each image pixel. In addition, using a two cycle thresholding process in the proposed method leads to an automatic segmentation which would not need to a prior knowledge about number of clusters. The efficiency and robustness of the proposed approach in segmenting both medical and natural images on different type and range of noise has been 

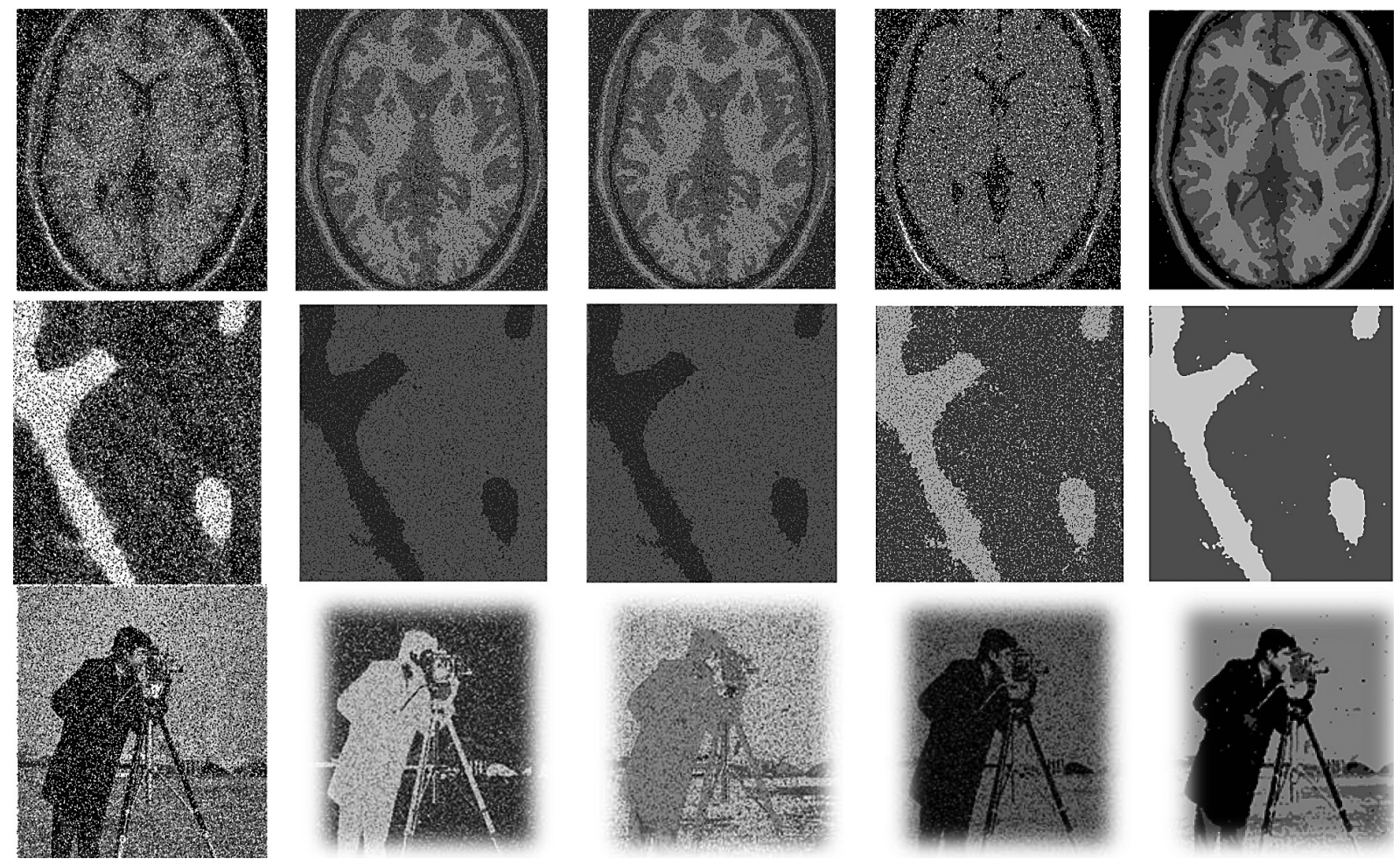

Fig. 5. The segmentation result of the three images contaminated with $25 \%$ salt and pepper noise in the first column was generated by the K-Mean (the second column), the FCM (the third column), the standard SOM (the fourth column) and the proposed SOM (fifth column)
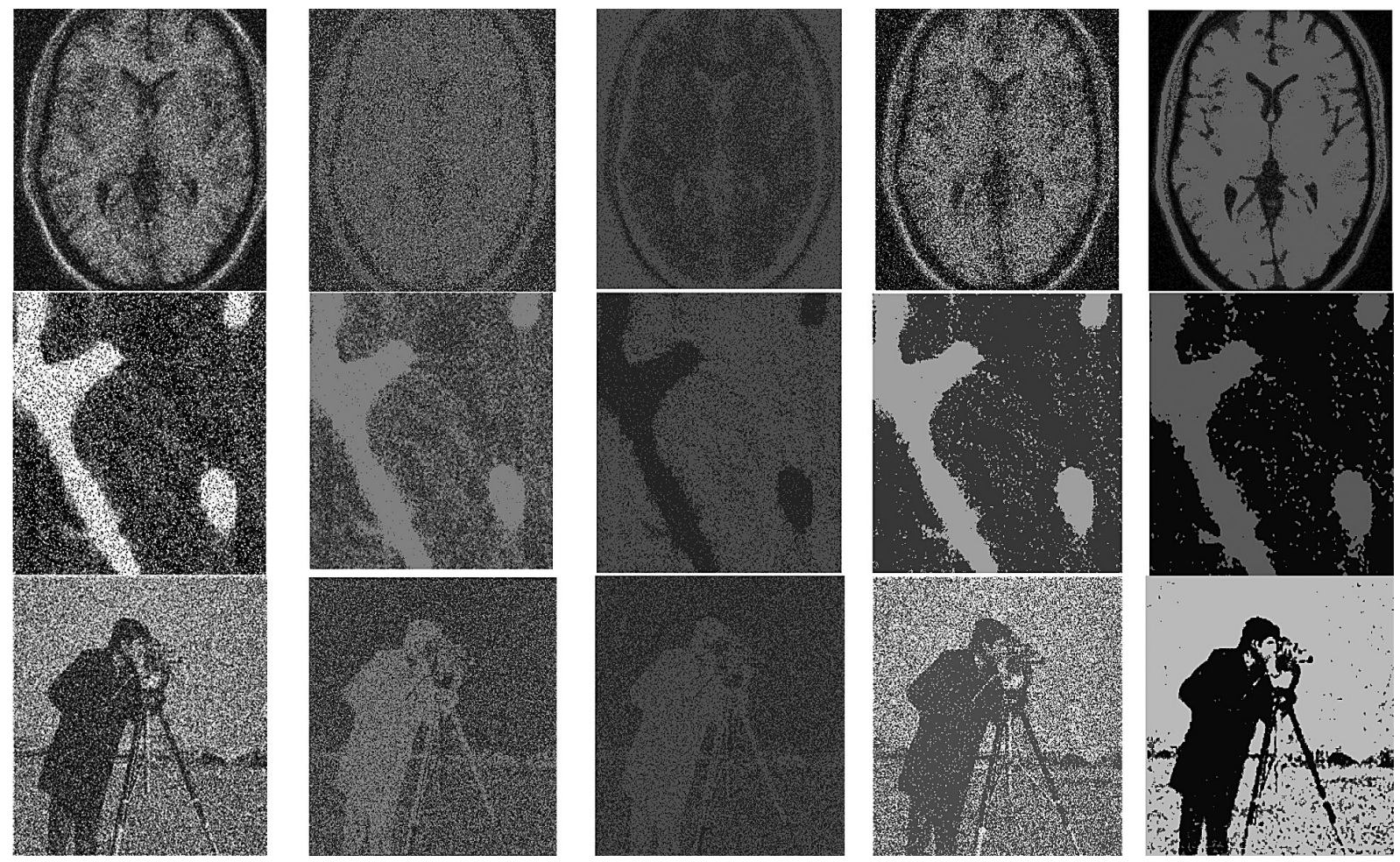

Fig. 6. The segmentation result of the three images contaminated with $25 \%$ Gaussian noise in the first column was generated by the K-Mean (the second column), the FCM (the third column), the standard SOM (the fourth column) and the proposed SOM (fifth column) 
demonstrated using experimentation with several images corrupted with salt \& pepper and Gaussian noises.

\section{REFERENCES}

1. Pratt W., John Wiley \& Sons, New York 1991.

2. Awad M., An unsupervised artificial neural network method for satellite image segmentation. The International Arab Journal of Information Technology, vol. 7, April 2010.

3. Awad M., Nasri A., Satellite image segmentation using self-organizing maps and fuzzy C-Means. IEEE, 2009, 398-402.

4. Jesna M., Raimond K., A survey on MR Brain image segmentation using SOM based strategies. International Journal of Computational Engineering Research, vol. 3, 2013.

5. Lopes A., Nezry E., Touzi R., Laur H., Maximum a posteriori speckle filtering and first order textural models in SAR images. [In:] Proceedings of International Geoscience and Remote Sensing Symposium, Maryland, 1990, 2409-2412.

6. Benediktsson J., Swain P., Ersoy O., Hong D., Neural network approaches versus statistical methods in classification of multisource remote sensing data. Computer Journal of Institute of Electrical and Electronics Engineers, 28(4), 1990, 540-551.

7. Perkins S., Theiler J., Brumby S., Harvey N., Porter R., Szymanski J., Bloch J., GENIE: A hybrid genetic algorithm for feature classification in multi spectral images. [In:] Proceedings of SPIE Applications and Science of Neural Networks, Fuzzy Systems and Evolutionary Computation III 4120, USA, 2000, 52-62.

8. Zhang P., Verma B., Kumar K., Neural vs statistical classifier in conjunction with genetic algorithm feature selection in digital mammography. IEEE, 2003.

9. Zhou Z., Wei S., Zhang X., Zhao X., Remote sensing image segmentation based on self organizing map at multiple scale. Proceedings of SPIE Geoinformatics: Remotely Sensed Data and Information, USA, 2007.

10. Kohonen T., Self-organizing maps. Computer Journal of Springer Series in Information Sciences 30(3), 2001, 501-505.
11. Wirjadi O., Survey of $3 \mathrm{~d}$ image segmentation methods. 2010.

12. Tian D., Fan L. et al. MR images segmentation method based on SOM neural network. [In:] Proceedings of the First International Conference on Bioinformatics and Biomedical Engineering, ICBBE, China, 2007, 686-689.

13. Kohonen T., Kaski S., Lagus K., Salojarvi J., Honkela J., Paatero V., Saarela A., Self organization of a massive document collection. IEEE Transactions on Neural Networks, vol. 11, 2000, 574-585.

14. Yeo N.C., Lee K.H., Venkatesh Y.V., Ong S.H., Colour image segmentation using the self-organizing map and adaptive resonance theory. Elsevier, 2005.

15. Demirhan A., Guler I. Combining stationary wavelet transform and self-organizing maps for brain MR image segmentation. Engineering Applications of Artificial Intelligence, vol. 24, 2011. 358-367.

16. Li Y., Chi Z., MR Brain image segmentation based on self-organizing map network. International Journal of Information Technology, vol. 11, 2005, 45-53.

17. Available insight segmentation and registration toolkit (ITK), an open source and cross platform system: http://www.itk.org/.

18. Alipour S., Shanbehzadeh J., Fast automatic medical image segmentation based on spatial kernel fuzzy c-means on level set method. Machine Vision and Applications, vol. 25, 2014, 1469-1488.

19. Gilboa G., Osher S., Nonlocal linear image regularization and supervised segmentation. SIAM Multiscale Modeling and Simulation, vol. 6, 2007, 595-630.

20. Nadernejad E., Sharifzadeh S., A new method for image segmentation based on Fuzzy C-means algorithm on pixonal images formed by bilateral filtering. Signal Image Video Process, vol. 7, 2013, 855-863.

21. Bernard O., Friboulet D., Thévenaz P., Fellow M., Variational B-spline leve 1-set: a linear filtering approach for fast deformable model evolution. IEEE Trans. Image Process, vol. 18, 2009, 1179-1191.

22. Jain A., Dubes R., Algorithms for clustering data. Englewood Cliffs, NJ: Prentice-Hall, 1988.

23. Bezdek J.C., Pattern recognition with fuzzy objective function algorithms. Plenum Press, New York, 1981. 